\title{
FMRI Decoding of Intentions: Compositionality, Hierarchy and Prospective Memory
}

\author{
John-Dylan Haynes, David Wisniewski, Kai Görgen, \\ Ida Momennejad \\ Bernstein Center for Computational Neuroscience \\ Charité - Universitätsmedizin Berlin \\ Charitéplatz 1 - 10117 Berlin - Germany \\ haynes@bccn-berlin.de
}

\author{
Carlo Reverberi \\ Department of Psychology \\ Università Milano-Bicocca \\ Piazza dell'A teneo Nuovo, 1 - 20126 Milan - Italy \\ carlo.reverberi@unimib.it
}

\begin{abstract}
In recent years multivariate decoding has allowed to test where and how mental representations can be decoded from neuroimaging signals, which sheds light on how these representations are encoded in the brain. In one line of experiments, we investigated how intentions are encoded in fMRI signals, thus revealing information in medial and lateral prefrontal regions. These informative neural representations were even present prior to the person's awareness of their chosen intention. In comparison, for cued intentions we found information predominantly in lateral, but not medial prefrontal cortex. Intention coding in prefrontal cortex followed a compositional code and could also be observed across extended delays during which participants were busy performing other tasks. Taken together, our results suggest a systematic, compositional and hierarchical code in prefrontal cortex which intentions are encoded across delays while the mind is busy working on other tasks.
\end{abstract}

Keywords-intention, task set, prospectiv memory, Libetexperiment, fMRI, multivariate decoding

\section{INTRODUCTION}

In recent years substantial progress has been in understanding the neural code in which intentions are encoded in the human and primate brains (e.g. [1], [2], [3], [4]). An important line of research was to decode intentions from neuroimaging signal patterns obtained while humans prepare for specific tasks. For free chosen intentions, this revealed information in medial (and to a lesser degree in lateral) prefrontal cortex [5]. For cued intentions, information was primarily observed in lateral prefrontal and parietal cortices [6]. For freely chosen intentions, it was even possible to predict a person's choice for a simple motor actions as well as complex cognitive tasks to some degree already several seconds before they made up their mind ([7], [8], [9], [10]). Here we report on several lines of research that have used classifiers to shed a more detailed light on the nature of intention representations in the human brain.

\section{COMPOSITIONALITY}

In everyday life we often use rules to organize action and thoughts for reaching our goals. Sometimes it is enough to make use of extremely simple rules, just linking a specific sensory pattern (e.g. "dad talks") to a consequent action (e.g "listen carefully"). However, in most real life scenarios, it is necessary to use more complex rule sets, which may require, for example, the concurrent application of multiple simple rules [3]. Previous evidence on humans and monkeys has shown that a fronto-parietal network is involved during rule maintenance and processing ([1], [2]). Nevertheless, the fundamental question of how the human brain represents more complex rules was left unexplored. In this experiment [11] we asked whether the neural code used by the human brain to represent "compound" rule sets is compositional: is the neural code of a rule set composed by rule A plus rule $\mathrm{B}$ built upon the neural representation of rule $\mathrm{A}$ and rule $\mathrm{B}$ when considered alone? Subjects were asked to first maintain and then evaluate simple (e.g. "If there is a face press left"), or compound rules (e.g. "If there is a face press left" combined with "If there is a house press right"). Multivariate pattern analysis (MVPA) was applied to functional MRI data only related to the pure maintenance phase of the task. We found that both right ventrolateral prefrontal cortex (mostly Brodmann Area 47) and left intraparietal sulcus (BA 40) encoded the active compound rule during maintenance. Most importantly however, we showed that in ventrolateral prefrontal cortex, but not in parietal cortex, it was possible to decode the active compound rule by only relying on the neural patterns associated to the simple rules. This shows that brain uses a compositional code in ventrolateral PFC.

\section{RULE ORDER}

Besides rules linking sensory evidence to action, another elementary building block of flexible "mental programs" [12] is order. Order may be conceived as an additional information layer specifying the sequence in which rules should be evaluated. In a further experiment [13], we considered rule sets not only comprising multiple rules, but also containing explicit instructions on which of the composing rules should be evaluated first. The experiment aimed at clarifying whether the human brain encodes the rule identity and the order in the same "rule-set" region, or rather it segregates and distributes relevant information in different areas. Participants had to maintain and evaluate two compound rules very similar to those used in the preceding experiment, e.g. "If there is furniture press letter A" combined with "If there is a fruit press letter B". Importantly, 
one of the two rules had to be evaluated first: in some trials the "furniture" rule, in other the "fruit" rule.

A
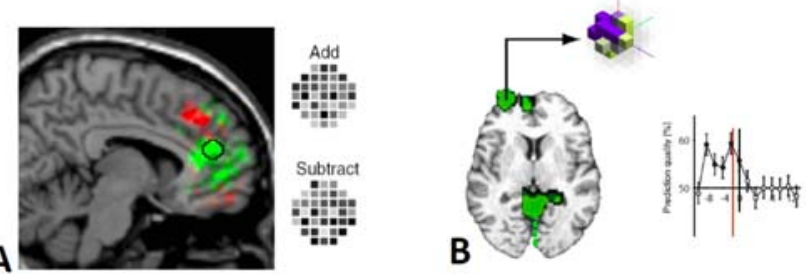

C

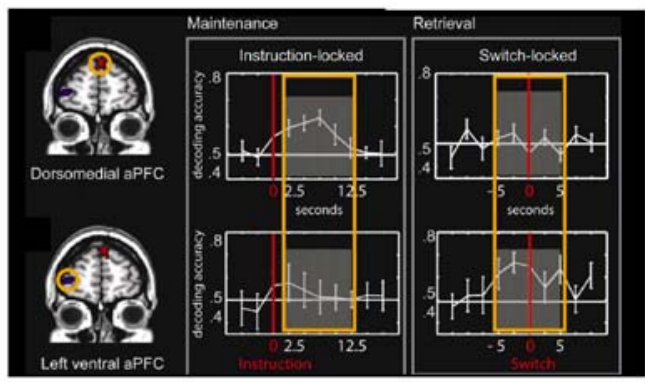

Figure 1: Decoding of intentions from human fMRI signals. (A) Decoding of a self-chosen intention during a post-decision delay [5]. (B) Predictive decoding of a freely chosen intention several seconds before a person believes to be making up their mind. (C) Encoding of prospective intentions during maintenance period and retrieval period [14].

We applied multivariate pattern analysis to the fMRI signal related to the maintenance phase independently assessing the neural representation of rule identity and rule order. We found that rule identity was encoded in a network including the right ventrolateral prefrontal cortex. This directly replicates the finding on compound rule representation of the preceding study. By contrast, rule order was encoded in dorsal striatum and right premotor cortex. Most importantly, we found a region $\mathrm{x}$ information interaction, formally showing a dissociation between areas representing identity and those representing rule order. Together with the previous study [11], these results strongly suggest that the human brain independently encodes the elementary features of complex rule-sets. Those elementary features are then represented in brain structures appropriate for the type of information. More in general, these studies open the possibility of understanding how our brain represents complex actions plans by learning the neural patterns of the known composing elements and how they are integrated.

\section{DELAY: ProspeCtive INTENTIONS}

The prospective remembering of planned actions is central to the success of goal-directed behavior ([15], [16]). Prospective memory relies on the capacity to perform the intended task either at (a) an intended point in time, i.e. timebased prospective memory ([14], [17]) or (b) at the appearance of the relevant event or cue, i.e. event-based prospective memory ([18], [19]).

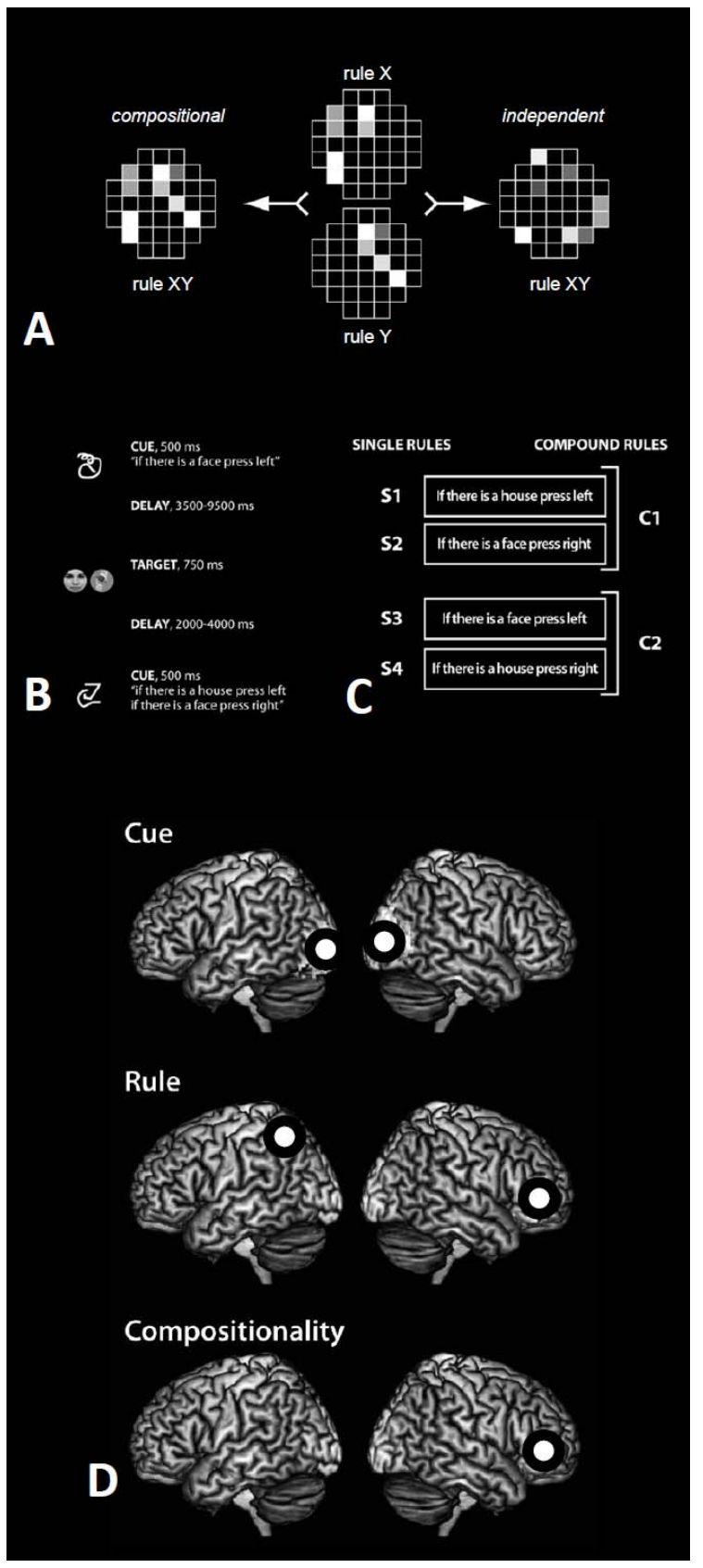

Figure 2: Compositionality of rule encoding in prefrontal but not parietal cortex [11]. (A) Compositional versus noncompositional codes. (B,C): Cued intention task with either simple or complex rules. (D) Decoding of cues, rules and compositional rules.

Importantly, intermediate tasks between the formation and execution of intended actions share working memory and attentional resources. How does the brain maintain future plans and monitoring the environment for the right time of action? In two studies using cued intentions, we combined behavioral experiments in prospective memory and fMRI and showed that it is possible to decode the subject's intended action (a) from the rostrolateral PFC during retrieval of time-based PM [14] and during the maintenance of delayed intentions during a 
delay whether it was filled with another task or not [19]; and (b) from the rostromedial PFC (medial aPFC) during the formation and early maintenance of of time-based PM during an occupied delay [14] and during the maintenance of a delayed intention during a delay occupied with another task, or event-based PM [19]. In a comparison of a number of MVPA studies on prospective memories, we have shown that our findings fit with a general function for rostrolateral PFC and an intention-dependent function along rostrocaudal axis of the medial PFC in the representation of delayed intentions. More rostral or anterior medial PFC regions are more likely to engage in the formation of self-initiated intentions or the maintenance of delayed intentions during occupied delays whereas more posterior medial PFC regions were involved in un-filled delays [19].

\section{FORAGING}

While freely forming intentions, we have to balance different competing demands. On the one hand, we should flexibly switch away from a currently performed task, e.g. if the reward outcome is too low or the difficulty is too high [20]. On the other hand, we should avoid frequent switching between different tasks, as this carries a cost in itself [21]. Recently, we developed a free choice task that allowed us to assess the neural representation of intentions that were motivated by dynamically and predictably changing difficulty conditions [22]. Subjects could freely choose between three different tasks in each trial. If they chose the same task repeatedly, the difficulty increased. If they abstained from choosing a task for some time, the difficulty of this task decreased. These rules were known to the subjects, the environment thus reacted to their decisions predictably. Using multivariate decoding, we were able to identify a region in the right dorsomedial prefrontal cortex and dorsal anterior cingulate cortex (dmPFC/dACC) that encoded subjects' intentions to perform a specific task. In a second, independent decoding analysis, we also identified a wide-spread brain network which represented the task difficulty, i.e. the main variable motivating task choices. Critically, the $\mathrm{dmPFC} / \mathrm{dACC}$ was the only brain region that encoded both the task choice and the task difficulty independently [23].

\section{ACKNOWLEDGMENT}

This work was supported by the Bernstein Computational Neuroscience Program of the German Federal Ministry of Education and Research (grant reference 01GQ1001C) and the Deutsche Forschungsgemeinschaft (DFG) Grant SFB 940. It was further supported by DFG grants Exc 257 Neurocure and KFO247. C.R. was also supported by the PRIN grant 2010RP5RNM_001 from the Italian Ministry of University.

\section{REFERENCES}

[1] J.D. Wallis, K.C. Anderson, E.K. Miller, "Single neurons in prefrontal cortex encode abstract rules" Nature, vol 411, pp. 953-956, 2001.

[2] K. Sakai, R.E. Passingham, "Prefrontal interactions reflect future task operations" Nature Neuroscience, vol. 6, pp. 75-81, 2003.
[3] S.A. Bunge, J.D. Wallis, „Neuroscience of rule-guided behavior” Oxford University Press, 2007

[4] M.F.S. Rushworth, M.P. Noonan, E.D. Boorman, M.E. Walton, T.E. Behrens, "Frontal cortex and reward-guided learning and decisionmaking” Neuron, vol. 70, pp. 1054-1069, 2011.

[5] J.D. Haynes, K. Sakai, G. Rees, S. Gilbert, C. Frith, R.E. Passingham, "Reading hidden intentions in the human brain" Curr Biol, vol. 17, pp. 323-328, 2007.

[6] S. Bode, J.D. Haynes, "Decoding sequential stages of task preparation in the human brain" Neuroimage, vol. 45, pp. 606-613, 2009.

[7] S. Bode, A.H. He, C.S. Soon, R. Trampel, R. Turner, J.D. Haynes, "Tracking the unconscious generation of free decisions using ultra-high field fMRI" PLoS One, vol. 6, e21612, 2011.

[8] C.S. Soon, M. Brass, H.J. Heinze, J.D. Haynes, "Unconscious determinants of free decisions in the human brain" Nat Neurosci, vol. 11, pp. 543-545, 2008.

[9] C.S. Soon, A.H. He, S. Bode, J.D. Haynes, "Predicting free choices for abstract intentions" Proc Natl Acad Sci USA, vol. 110, pp. 6217-6222, 2013.

[10] C.S. Soon, C. Allefeld, C. Bogler, J. Heinzle, J.D. Haynes, „Predictive brain signals best predict upcoming and not previous choices" Front Psychol, vol. 8, pp. 406, 2014.

[11] C. Reverberi, K. Görgen, J.D. Haynes, „Compositionality of rule representations in human prefrontal cortex" Cerebral cortex, vol. 22, pp. 1237-1247, 2012.

[12] J. Duncan, "The multiple-demand (MD) system of the primate brain: mental programs for intelligent behaviour". Trends Cogn Sci, vol. 14, pp. 172-179, 2010.

[13] C. Reverberi, K. Görgen, J.D. Haynes, "Distributed representations of rule identity and rule order in human frontal cortex and striatum" $\mathrm{J}$ Neurosci, vol. 32, pp. 17420-17430, 2012.

[14] I. Momennejad, J.D. Haynes, "Human anterior prefrontal cortex encodes the 'what' and 'when' of future intentions" Neuroimage, vol. 61, pp. 139-148, 2012.

[15] M.A. Brandimonte, G.O. Einstein, M.A. McDaniel, "Prospective memory: theory and applications". Erlbaum, Hillsdale, NJ, 1996.

[16] P.W. Burgess, G. Gonen-Yaacovi, E. Volle, "Functional neuroimaging studies of prospective memory: what have we learnt so far?" Neuropsychologia, vol. 49,pp. 2246-2257, 2011.

[17] J. Okuda, T. Fujii, H. Ohtake, T. Tsukiura, A. Yamadori, C.D. Frith, P.W. Burgess, "Differential involvement of regions of rostral prefrontal cortex (Brodmann area 10) in time- and event-based prospective memory" Int J Psychophysiol, vol. 64, pp. 233-246, 2007.

[18] S.J, Gilbert, "Decoding the content of delayed intentions" J Neurosci, vol. 31, pp. 2888-2894, 2011.

[19] I. Momennejad, J.D. Haynes, "Encoding of prospective tasks in the human prefrontal cortex under varying task loads" J Neurosci, vol. 33, pp. 17342-17349, 2013.

[20] N.D. Daw, J.P. O’Doherty, P. Dayan, B. Seymour, R.J. Dolan, “Cortical substrates for exploratory decisions in humans" Nature, vol. 441, pp. 876-879, 2006.

[21] S. Monsell, "Task switching” Trends Cogn Sci, vol. 7, pp. 134-140, 2003.

[22] D. Wisniewski, C. Reverberi, A. Tusche, J.D. Haynes, "The neural representation of voluntary task-set selection in dynamic environments" Cer Cortex, bhu155, 2014.

[23] L. Pessoa, "How do emotion and motivation direct executive control?" Trends Cogn Sci, vol. 13, pp. 160-166, 2009. 